\title{
Research and Implementation of License Plate Character Recognition Technology Based on Neural Network
}

\author{
Chong $\mathrm{Xu}^{1, \mathrm{a}^{*}}$, Xin $\mathrm{Liu}^{2, \mathrm{~b}}$, Wei Wang ${ }^{3, \mathrm{c}}$,Yong Shi ${ }^{4, \mathrm{~d}}$ and Ning Tao ${ }^{5, \mathrm{e}}$ \\ ${ }^{1}$ BIM Computing Research Center, Shenyang jianzhu University, Shengyang, 110168, China \\ ${ }^{2}$ BIM Computing Research Center, Shenyang jianzhu University, Shengyang, 110168, China \\ ${ }^{3}$ BIM Computing Research Center, Shenyang jianzhu University, Shengyang, 110168, China \\ ${ }^{4}$ BIM Computing Research Center, Shenyang jianzhu University, Shengyang, 110168, China \\ ${ }^{5}$ BIM Computing Research Center, Shenyang jianzhu University, Shengyang, 110168, China \\ a32500103@qq.com, b620274@qq.com, b519836646@qq.com, d674365154@qq.com, \\ e23010150@qq.com \\ * The Corresponding Author
}

\begin{abstract}
Keywords: Plate recognition; Neural network; Character matching
Abstract. This paper studies the problem of character recognition in real time dynamic vehicle, and puts forward a new method to combine the improved neural network technology with the improved template matching method. Based on image boundary segmentation technology, based on vertical projection method, the character segmentation method is divided accurately, then the character recognition is realized by character network feature method, and the result is output character recognition. The experimental results show that the improved algorithm improves the real time of the system while ensuring the accuracy of license plate matching.
\end{abstract}

\section{Introduction}

With the development of traffic network and the development of computer video pattern recognition technology, the license plate recognition system has the characteristics of high adaptability and intelligence. As the key link in the intelligent transportation system, it is also one of the important challenges in the intelligent transportation field to realize the real-time, accurate and efficient license plate recognition system and ensure the traffic safety and intelligent management under large data conditions [1]. plays a key role in the intelligent monitoring and management of vehicles in the transportation system [2].

\section{Design and Implementation of Algorithm}

Plate Character Segmentation. Interference elements also contain 4 boundaries of vehicle license plates during character segmentation after image location. The boundary distribution is regular and it is easy to remove this interference factor. The number of white dots per line should be added to the array $C 1[i] \mathrm{In}$, then on $C 1[i]$ Translation, translation radius for the image height of 1 square 10; When a license plate image is projected vertically, the number of white dots per line should be added to the array $C 2[i] \mathrm{In}$, then on $\mathrm{C} 2[i]$ Translation, translation radius for the image height of 1/ 30.

Character Segmentation based on Vertical Projection. In the horizontal projection, the image area is measured to determine whether there is a space for storing characters in the area to be identified and the columns of these characters are combined so that the resulting character area is valid. Setting $\boldsymbol{K}$ as a one-dimensional vector is equal to the length $\boldsymbol{W}$ of the vehicle license plate. The sum of the bright spots in each pixel in the image is represented by the values in the vector. The minimum value of the projection of the block in the vertical direction is obtained in the space between each character and each character. From the figure, it is clear that there are gaps in the projection, of which 5 are between 6 characters and the remaining 3 are between [3].

The data columns are grouped into six groups and mapped to six separate arrays. In character 
recognition mode, the template size of each character varies, the pattern is different, and the resulting 6 arrays need to be transformed into matrix size, so that each changed array can reach $26 \times 14$. Segmentation of 6 characters of license plate image by image location.

Character Segmentation based on Neural Networks . Neural network pattern recognition is an important research direction and research content in the field of pattern recognition. It is a recognition method based on neural network pattern. It can realize the processing of pattern information which can not be finished by pattern recognition theory. If the sum of the signals received by a neuron exceeds its "threshold", the neuron is excited and sends a pulse to the next neuron.[4] . Through the network system of neurons and axons, we can approach any rational function and accomplish various simple or complicated tasks.

Neurons are relatively simple in structure and function, and the neural network is made up of a large number of simple basic units, which make up a nonlinear dynamic system. In the process of pattern recognition, there is some loss of noise interference or input mode, and artificial neural network can store information effectively in the weight value of the connection, so that the neural network has strong and fault tolerance. Each neuron has a lot of capacity for nonlinear mapping, and they are interconnected by weights. Artificial neural network(ANN) has the function of self-organization and adaptive learning, which greatly reduces the constraints necessary for traditional recognition methods, and makes it prominent in some identification problems compared with traditional machines.

Reverse propagation(BP) model is the most studied and applied ANN model. BP neural network structure is a multilayer network which trains weights by using error response propagation algorithm. The theory has proved that a 3-layer BP network model can realize arbitrary continuous image.

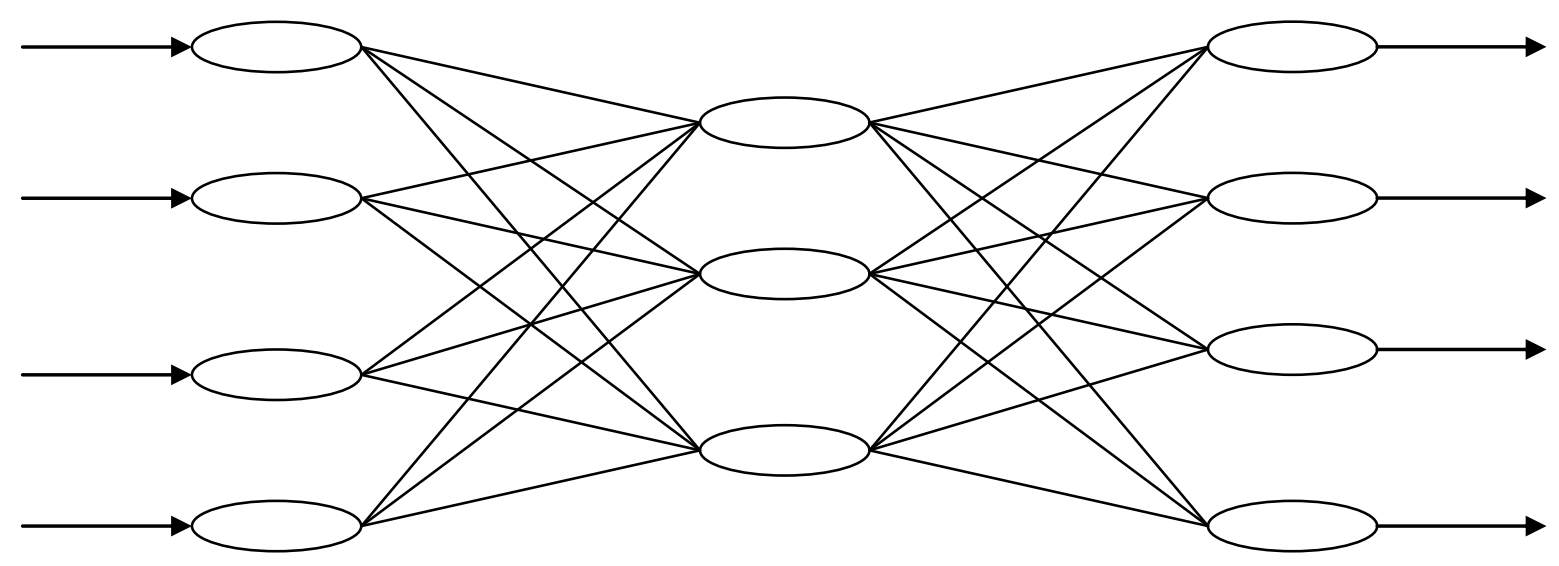

Input layer Hidden layer Output layer

Figure 1. Back propagation (BP) neural network structure

License Plate Character Recognition Method Template Matching Template matching method is typical in many kinds of license plate character recognition methods. The method has a large amount of computation, low accuracy and accuracy. By comparing the characters to be recognized with the ones already set, the result of character recognition is determined according to their matching similarity. The character image to be identified is $I$, the size of which is $W \times H$, the size of which is $M \times N$ and $W>M, H>N$. The matching process is to superimpose the template $\mathrm{T}$ on the character image $I$, and then analyze the subgraphs obtained by comparing the template $T$ with the character image $I$ If the difference between them is less than the default threshold, you can determine that template $T$ matches the subgraph of character image $I$.

The first item is the autocorrelation of a subset of character image $I$. The second item is the 
autocorrelation of character template $T$. The third item indicates that the greater the correlation between the first two items, the smaller the value of $D(i, j)$, and the better the match between the subgraph of character image I and character template $T . D(i, j)=0$ indicates that the similarity between character image I and character template is $100 \%$, which is a full batch match. Template matching by image processing equipment is relatively simple and less affected by noise, so it is often used in vehicle license plate character recognition, but it also has many disadvantages. For example: data computation is large, the number of operations to identify matching acquaintance will be more and more due to the increase of the size of the image to be identified, resulting in the real time efficiency of matching is reduced ; If the image processing of the character to be recognized has reflection transform, it will reduce the accuracy of template matching method.

Neural Network Identification. Neural network recognition pulse neural network processing of spatial and temporal information, the construction of the basic framework and evaluation of performance, including the learning ability of sequence pulses, the local characteristics of learning rules, adaptability to network structure, learning accuracy. It consists of two kinds of situations: one is to send the whole image into the network, and use the characteristics of the neural network to extract and recognize character characteristics from the dynamic realization; The second is to extract character features from recognition characters, then pass them to neural network classifier, and then enter them into neural network trainer with the weights of character features extracted from character sample after BP network training.

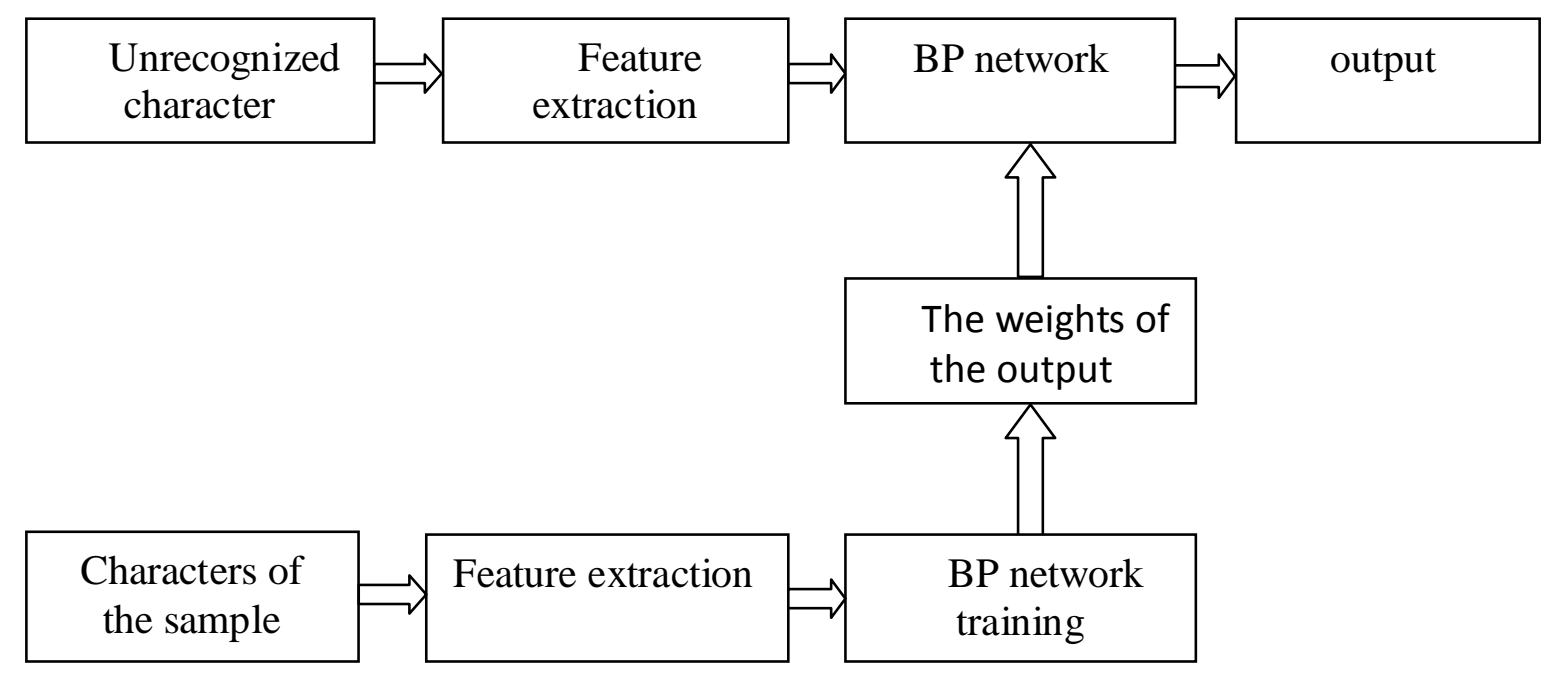

Figure 2. Schematic diagram of typical character recognition of BP network

\section{Test and Analysis}

In order to verify the performance of the algorithm and find out the difference between the function requirement and the system, the dynamic real-time video is selected randomly to test the recognition rate of the whole system. Three sections of AVI video with $90 \mathrm{~min}, 30 \mathrm{~min}$ and $30 \mathrm{~min}$ were selected for this experiment, including : Conditions include a video of 90 min taken by a camera set up at a traffic stop with good lighting conditions and high traffic. In the light conditions are good, the camera is in the driving car, the installation position is not fixed, the image of the shaking of the case of video $30 \mathrm{~min}$; In the late afternoon, when light conditions were poor, the human eye felt the gray environment of the video taken $30 \mathrm{~min}$. Among them, the video of $90 \mathrm{~min}$ included 720 vehicles with visible plates in good lighting, the video of $30 \mathrm{~min}$ installed in the vehicle contained 85 vehicles with vehicle identification plates in person and 160 vehicles with vehicle identification plates in poor lighting conditions.

During the experiment, 6,300 vehicles were identified in $90 \mathrm{~min}$ videos with good lighting conditions, 69 vehicles were identified in the video mounted in the vehicle with $30 \mathrm{~min}$, and 125 vehicles were identified under poor lighting conditions. The results of the experiment are shown in 
Table 1 Dynamic video test results

\begin{tabular}{|c|c|c|c|c|c|c|c|}
\hline $\begin{array}{l}\text { External } \\
\text { environme } \\
\text { nt }\end{array}$ & $\begin{array}{l}\text { Positionin } \\
\mathrm{g} \\
\text { accuracy } \\
\%\end{array}$ & $\begin{array}{l}\text { Vehicl } \\
\text { e } \\
\text { licens } \\
\text { e error } \\
\text { rate } \%\end{array}$ & $\begin{array}{l}\text { Vehicle } \\
\text { detectio } \\
\mathrm{n} \\
\text { rate } \%\end{array}$ & $\begin{array}{l}\text { Character } \\
\text { recognitio } \\
\text { n rate }\end{array}$ & $\begin{array}{l}\text { Segmen } \\
\mathrm{t} \text { - ation } \\
\text { accurac } \\
\mathrm{y} \%\end{array}$ & $\begin{array}{l}\text { Recognition } \\
\text { speed } \\
\text { (frame / } \\
\text { seconds) }\end{array}$ & $\begin{array}{l}\text { Overall } \\
\text { recognit } \\
\text { ion rate } \\
1 \%\end{array}$ \\
\hline $\begin{array}{l}\text { Light is in } \\
\text { good } \\
\text { position }\end{array}$ & 97.6 & 1.3 & 91.8 & 93.8 & 97.8 & 25 & 89.6 \\
\hline $\begin{array}{l}\text { Light is in } \\
\text { good } \\
\text { position. }\end{array}$ & 94.8 & 1.9 & 79.6 & 92.4 & 97.6 & 26 & 82.5 \\
\hline $\begin{array}{l}\text { Light is not } \\
\text { well fixed }\end{array}$ & 95.8 & 2.2 & 81.9 & 91.2 & 95.5 & 25 & 81.2 \\
\hline Combined & 96.1 & 1.8 & 84.4 & 85.1 & 96.9 & 25.3 & 84.4 \\
\hline
\end{tabular}

\section{Conclusion}

License Plate Recognition System (LPR) is a very important science technology in ITS. An algorithm based on improved neural network and improved template matching license plate character recognition is proposed. The effectiveness of the algorithm is proved by the experiment simulation. The advantage of the algorithm is to overcome the difficulty of template matching in finding the shape sub images of numbers, letters and characters.

\section{Reference}

[1] SHAN S G, KAN M N, LI S X, et al. Face image analysis and recognition with deep learning[J]. Communications of the CFF, 2015, 11(4):15-21.

[2] ZHU M, LIU C,WANG J, et al. Instant discovery of moment companion vehicles from big streaming traffic data [C].2015 International Conference on Cloud Computing and Big Data(CCBD), 2015:73-80.

[3] PRIYA V LAKSHMI, PERUMAL K. Detecting the car number plate using segmentation[J]. International Journal of Computer Science,2014(10):8823-8829.

[4] Using Artificial Neural Networks for Energy Regulation Based Variable-speed Electro hydraulic Drive[J]. Chinese Journal of Mechanical Engineering, 2010, 3:327-335. 